\title{
Inferior sinus venosus and two ostium secundum atrial septal defects is possible to treat with three occluders
}

Petr Hajek ${ }^{1}$, Jana Palenickova ${ }^{1}$, Jiri Fiedler ${ }^{1}$, Martin Horvath ${ }^{1}$, Vojtech Suchanek$^{2}$, Josef Veselka ${ }^{1}$

\author{
${ }^{1}$ Department of Cardiology, $2^{\text {nd }}$ Faculty of Medicine and Motol University Hospital, \\ Prague, Czech Republic \\ ${ }^{2}$ Department of Imaging Methods, $2^{\text {nd }}$ Faculty of Medicine and Motol University \\ Hospital, Prague, Czech Republic
}

Submitted: 26 November 2014

Accepted: 3 January 2015

Arch Med Sci 2017; 13, 1: 260-262

DOI: 10.5114/aoms.2016.64041

Copyright (c) 2016 Termedia \& Banach

A 73-year-old woman known to have multiple atrial septal defects (mASD) since 2006 rejected cardiac surgery and was referred to our department with signs of right heart failure. Echocardiography demonstrated one defect located in the inferior portion of the atrial septum that led to an overriding inferior vena cava (IVC) - an inferior sinus venosus defect (SVD) and two additional ostium secundum ASDs (ASDIl; Figure 1). Furthermore, a significant left-to-right atrial shunt and dilation of the right ventricle were observed.

Cardiac surgery was reconsidered but ultimately refused by the patient. During right heart catheterization, the mean pulmonary pressure was $28 \mathrm{~mm} \mathrm{Hg}$, and the pulmonary-to-systemic flow ratio was 4.0. Therefore, we proceeded with percutaneous closure of the mASD; three Figulla ASD occluders (Occlutech GmbH, Germany; $21 \mathrm{~mm}, 21 \mathrm{~mm}$ and $10 \mathrm{~mm}$ ) were successfully implanted (Figure 2). The pulmonary-to-systemic flow ratio after the mASD closure procedure decreased to 1.1. At a 1-month follow-up, the patient reported significant improvements in her symptoms, and adequate positioning of the occluders was demonstrated (Figure 2).

The percutaneous closure of ASDIIs has become the standard of care in the majority of patients [1]. Surgical correction is reserved for other types of ASD, such as ostium primum and sinus venosus ASDs. Inferior SVDs are rare ASDs that originate in the mouth of the IVC, continue directly into the infero-posterior border of the left atrium and leave no residual atrial septal tissue at the inferior margin [2].

To the best of our knowledge, this the first case of percutaneous closure of an inferior SVD with the simultaneous use of three Figulla occluders for the closure of an mASD. The successful result of our procedure suggests that, in patients who refuse surgery, the percutaneous approach should be considered. However, surgery remains the treatment of choice for inferior SVD closures.

\section{Acknowledgments}

This study was supported by the Ministry of Health of the Czech Republic and the conceptual development of research organization of the University Hospital Motol, Prague, Czech Republic 00064203.

\section{Conflict of interest}

The authors declare no conflict of interest.

\author{
Corresponding author: \\ Josef Veselka MD, PhD \\ Department of Cardiology \\ $2^{\text {nd }}$ Faculty of Medicine \\ and Motol University Hospital \\ V Uvalu 84 \\ Praha 5, 15000 Czech \\ Republic \\ Phone: +42 0224434901 \\ Fax: +42 0224434920 \\ E-mail: veselka.josef@ \\ seznam.cz
}



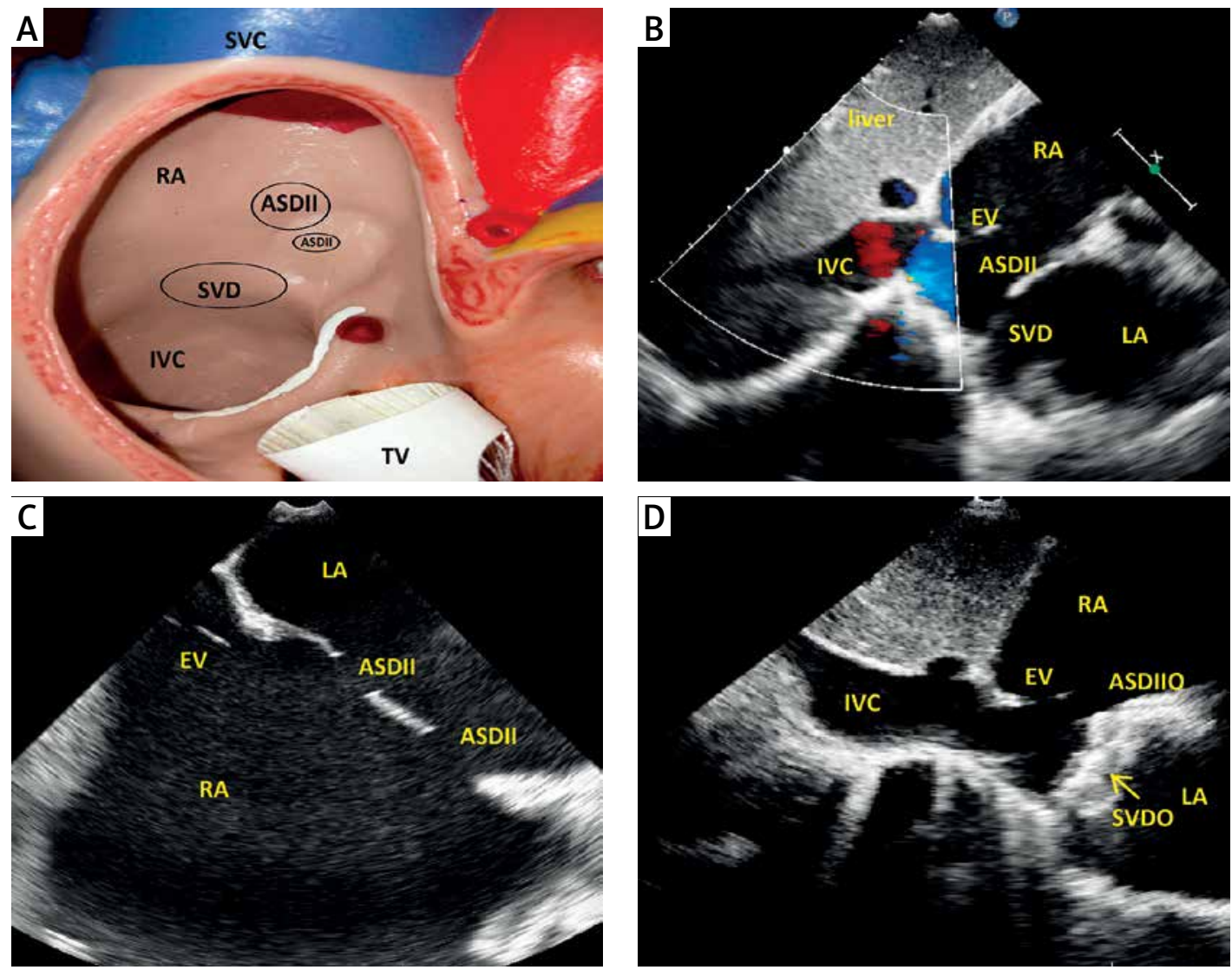

Figure 1. A - Schematic diagram of the mASD distribution of our case. B - Subcostal TTE showing the inferior SVD (the two ASDIls are not visible). C - TEE showing the two ASDIls (the inferior SVD is not visible). D - Subcostal TTE showing two occluders in a nearly rectangular configuration (the occluders in the inferior SVD and one of the ASDIls are shown; the occluder in the other ASDIl is not visible)
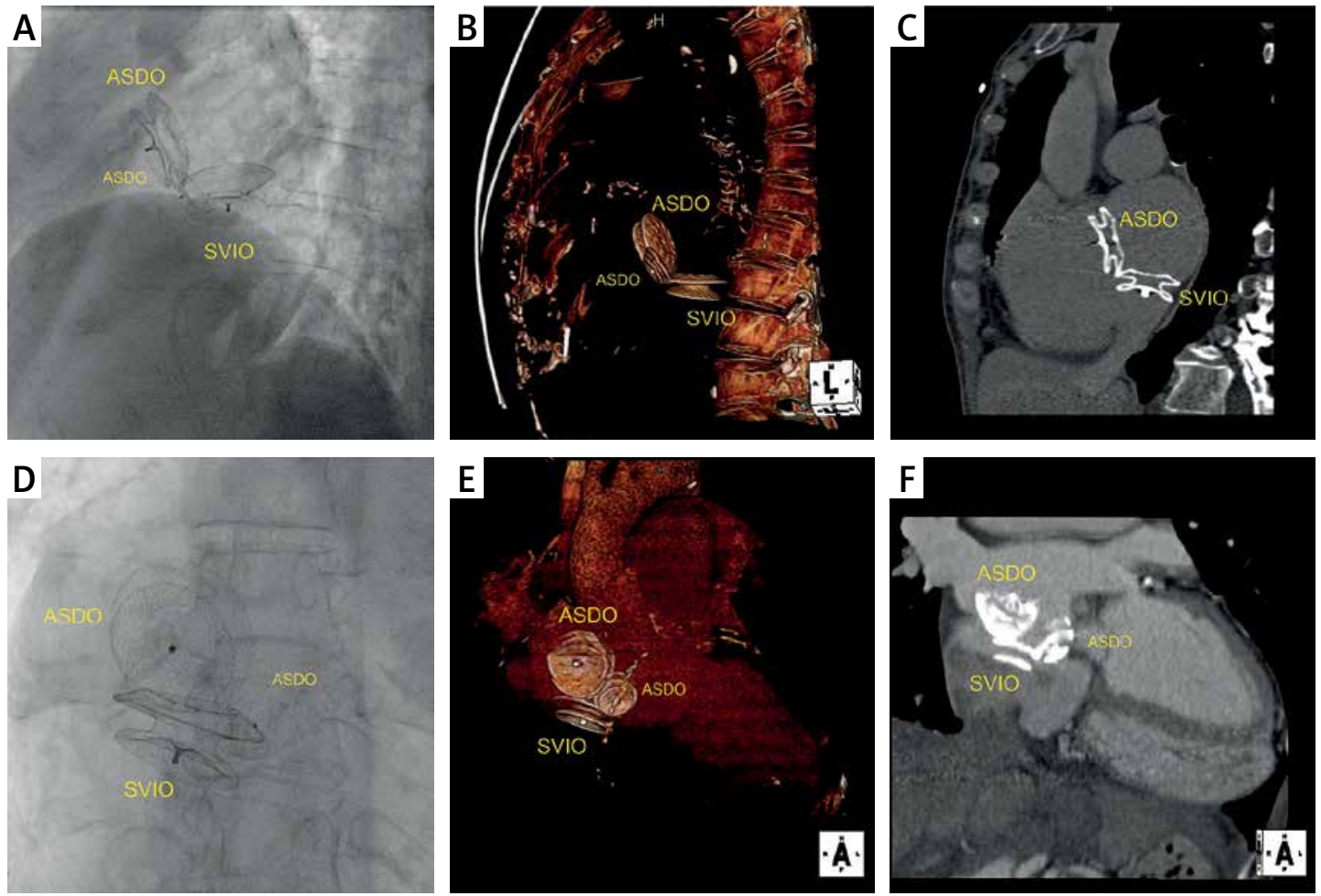

Figure 2. The three occluders as shown by fluoroscopy, three-dimensional CT reconstruction and two-dimensional $\mathrm{CT}$ in the lateral $(\mathbf{A}-\mathbf{C})$ and sagittal (D-F) views. Note the nearly rectangular configuration of the occluders in the SVD and one of the ASDIIs 


\section{References}

1. Tomasov P, Linhartova K, Antonova P, Adlova R, Alan D, Veselka J. Combined percutaneous treatment of atrial septal defect and pulmonic or aortic stenosis in adult patients. Arch Med Sci 2010; 6: 976-80.

2. Plymale J, Kolinski K, Frommelt P, Bartz P, Tweddell J, Earing MG. Inferior sinus venosus defects: anatomic features and echocardiographic correlates. Pediatr Cardiol 2013; 34: 322-6. 